\title{
Sub-picosecond Exciton Dynamics in Polyfluorene Films from Experiment and Microscopic Theory
}

\author{
Jean-Christophe Denis, ${ }^{\dagger}$ Stefan Schumacher ${ }^{\ddagger}$ Gordon J. Hedley, ${ }^{\llbracket}$ Arvydas \\ Ruseckas, " Paulina O. Morawska, "Yue Wang, " Sybille Allard, $§$ Ullrich Scherf, $\$$ \\ Graham A. Turnbull, "Ifor D.W. Samuel, ${ }^{*, \uparrow}$ and lan Galbraith ${ }^{*, \dagger}$ \\ Institute for Photonics and Quantum Sciences, School of Engineering and Physical \\ Sciences, SUPA, Heriot-Watt University, Edinburgh EH14 4AS, United Kingdom, Physics \\ Department and Center for Optoelectronics and Photonics Paderborn (CeOPP), Universität \\ Paderborn, Warburger Strasse 100, 33098 Paderborn, Germany, Organic Semiconductor \\ Centre, SUPA, School of Physics and Astronomy, University of St. Andrews, North Haugh, \\ St. Andrews, Fife KY16 9SS, United Kingdom, and Department of Chemistry and Institute \\ for Polymer Technology, Wuppertal University, 42097 Wuppertal, Germany
}

\section{Abstract}

Electronic energy transfer (EET) in organic materials is a key mechanism that controls the efficiency of many processes, including light harvesting antenna in natural and artificial photosynthesis, organic solar cells, and biological systems. In this paper we have examined EET in solid-state thin-films of polyfluorene, a prototypical conjugated polymer with ultrafast photoluminescence experiments and theoretical modelling. We observe EET occurring on a $680 \pm 300 \mathrm{fs}$ timescale by looking at the depolarisation of photoluminescence. An

${ }^{*}$ To whom correspondence should be addressed

$\dagger$ Institute for Photonics and Quantum Sciences, School of Engineering and Physical Sciences, SUPA, Heriot-Watt University, Edinburgh EH14 4AS, United Kingdom

${ }_{\ddagger}^{\ddagger}$ Physics Department and Center for Optoelectronics and Photonics Paderborn (CeOPP), Universität Paderborn, Warburger Strasse 100, 33098 Paderborn, Germany

IOrganic Semiconductor Centre, SUPA, School of Physics and Astronomy, University of St. Andrews, North Haugh, St. Andrews, Fife KY16 9SS, United Kingdom

${ }^{\S}$ Department of Chemistry and Institute for Polymer Technology, Wuppertal University, 42097 Wuppertal, Germany independent, predictive microscopic theoretical model is built by defining 125000 chromophores containing both spatial and energetic disorder appropriate for a spin-coated thin film. The model predicts time-dependent exciton dynamics, without any fitting parameters, using the incoherent Förster-type hopping model. Electronic coupling between the chromophores is calculated by an improved version of the usual line-dipole model for resonant energy transfer. Without the need for higher level interactions, we yet find that the model is in general agreement with the experimentally observed $680 \pm 300$ fs depolarisation caused by EET. This leads us to conclude that femtosecond EET in polyfluorene can be well described by conventional resonant energy transfer, as long as the relevant microscopic parameters are well captured. The implications of this finding are that dipole-dipole resonant energy transfer can in some circumstances be fully adequate to describe ultrafast EET without needing to invoke strong or intermediate coupling mechanisms. 


\section{Introduction}

A quantitative understanding of exciton migration in conjugated polymers is important for applications in organic photovoltaics, lightemitting diodes and lasers ${ }^{1-5}$. In polymer films excitons migrate along conjugated chains as well as between chains ${ }^{6-10}$. Recent experiments have shown that the coherence length of excitons along perfect and straight chains at cryogenic temperature can be several microns, so that the conjugated chain behaves like a quantum wire ${ }^{11}$. Even chains with conformational disorder can support significant delocalisation of primary excitons ${ }^{12,13}$. Förster energy transfer rates have been measured in fluorene-based blends, of PF8 and F8BT polymers ${ }^{14,15}$, with transfer times of over $10 \mathrm{ps}$, which is much longer than the decoherence time. Experiments also indicate ultrafast exciton localisation in $100 \mathrm{fs}$ in disordered polymers ${ }^{12,16}$ which can be explained by intra-band and structural relaxation ${ }^{12,16-18}$. It is not known which, if any, of these effects are generic for conjugated polymers and which are specific to particular chemical structures. Of particular interest to modern applications in photovoltaic and lightemitting devices are polymers with rigid repeat units including polyfluorene and its copolymers $^{19}$ as well as co-polymers with carbazole or benzodithiophene-diyl units ${ }^{2,20,21}$.

The combined influence of many factors, including sample morphology ${ }^{22,23}$, molecular conformations ${ }^{24-26}$, packing and traps ${ }^{27,28}$, exciton hopping and annihilation makes the problem of organic semiconductor device optimization complex, as it involves a wide range of energies, timescales and parameters that are difficult to isolate. Disentangling one from another is challenging and requires a combination of both experimental and theoretical approaches. Simulations of large-scale systems have been conducted using a Monte-Carlo simulation approach ${ }^{29-32}$ or a Master-equation based approach ${ }^{33-37}$, while experiments have used spectral- and time-resolved photoluminescence decay ${ }^{23,38,39}$ or transient absorption spectroscopy ${ }^{39,40}$ to monitor the ultrafast photophysics. Furthermore a number of theoreti- cal simulations model charge-separated excitations ${ }^{41-46}$, which are particularly relevant to current flow in devices such as photovoltaics and LEDs.

In this paper we measure ultrafast exciton dynamics and energy transfer in films of polyfluorene as a prototypical rigid rod polymer. Such quantitative knowledge of exciton dynamics is needed to predict, for example, the early time dynamics of solar generated excitations. The observed fast exciton migration can be well described by a predictive microscopic model which includes fast intra-molecular exciton localisation in $100 \mathrm{fs}$ followed by incoherent intermolecular hopping. Our results show that exciton dynamics in rigid-rod polymers can be successfully modelled using information on film density, steady state absorption and fluorescence spectra, and quantum chemical calculations. We demonstrate the merit of our approach in a direct experiment-theory comparison. We show that (i) ultrafast fluorescence depolarization on sub-picosecond timescales, (ii) exciton diffusivity, and (iii) exciton-exciton annihilation rates covering a wide range of excitation intensities, can all be quantitatively understood within our theoretical approach.

\section{Experiments}

\section{A. Methods}

Spin-coated (glassy-phase) polyfluorene (PF8, of molecular weights $M_{n}=135000 \mathrm{~g} / \mathrm{mol}$ and $M_{w}=299000 \mathrm{~g} / \mathrm{mol}$ ) films were excited by a $100 \mathrm{fs}$ (full-width half-maximum) laser pump pulse at an excitation wavelength of $400 \mathrm{~nm}$. The resultant time-resolved fluorescence dynamics polarized parallel $\left(I_{\|}\right)$and perpendicular $\left(I_{\perp}\right)$ to the excitation were measured at a detection wavelength of $475 \mathrm{~nm}$. From the measured fluorescence dynamics the polarisation anisotropy, $r(t)$, can then be reconstructed:

$$
r(t)=\frac{I_{\|}(t)-I_{\perp}(t)}{I_{\|}(t)+2 I_{\perp}(t)} .
$$

The polarisation-independent fluorescence dynamics (the so-called "magic angle") are de- 
fined by: $m(t)=\left(I_{\|}(t)+2 I_{\perp}(t)\right) / 3$. Further details on experimental methods are provided in the Supporting Information.

The instrument response function (IRF) was also recorded, and was found to have a 380 fs FWHM. Fitting of the polarisation anisotropy dynamics was performed using the impulse reconvolution method ${ }^{47}$ which enables deconvolution of the instrument response function from the decay. This requires convolving a sum of exponentials with the IRF and then fitting the results with the magic angle $m$ dynamics. The best-fit for the magic angle kinetics can then be convolved with a trial function representing the polarisation anisotropy decay and also convolved with the IRF. Iteration of the trial function to give a good fit to the numerator of equation (1) (the so-called "difference fit") enables the anisotropy to be fitted with full account taken of the IRF.

\section{B. Fluorescence Anisotropy Decay}

The ultrafast fluorescence kinetics in the polyfluorene film (PF8) on the red-side of the photoluminescence spectrum at $2.61 \mathrm{eV}$ are shown in figure 1(a). To the "magic angle" kinetics we fit fast and slow exponential risetimes of 200 fs and 2 ps, with 0.76 amplitude assigned to the fast component and 0.19 assigned to the slow, with the remaining 0.05 contribution instantaneously following the pump. On the red-side of the PL spectrum we observe an increase in PL intensity over the first few picoseconds, a rise attributed to electronic and geometry relaxation (e.g. bond lengths, torsional angles) and/or energy transfer between chromophores. To disentangle these processes from energy transfer between chromophores (on or between polymer chains), one must next consider the fluorescence anisotropy data, which provides information on the change in angle of the dipole moment of the exciton on an ultrafast timescale. The fluorescence anisotropy of PF8 at a detection wavelength of $475 \mathrm{~nm}$ is shown in figure $1(\mathrm{~b})$. We have chosen a detection wavelength on the red-side of the $\mathrm{PL}$ spectrum to ensure that there is absolutely no contamination of the recorded signal with
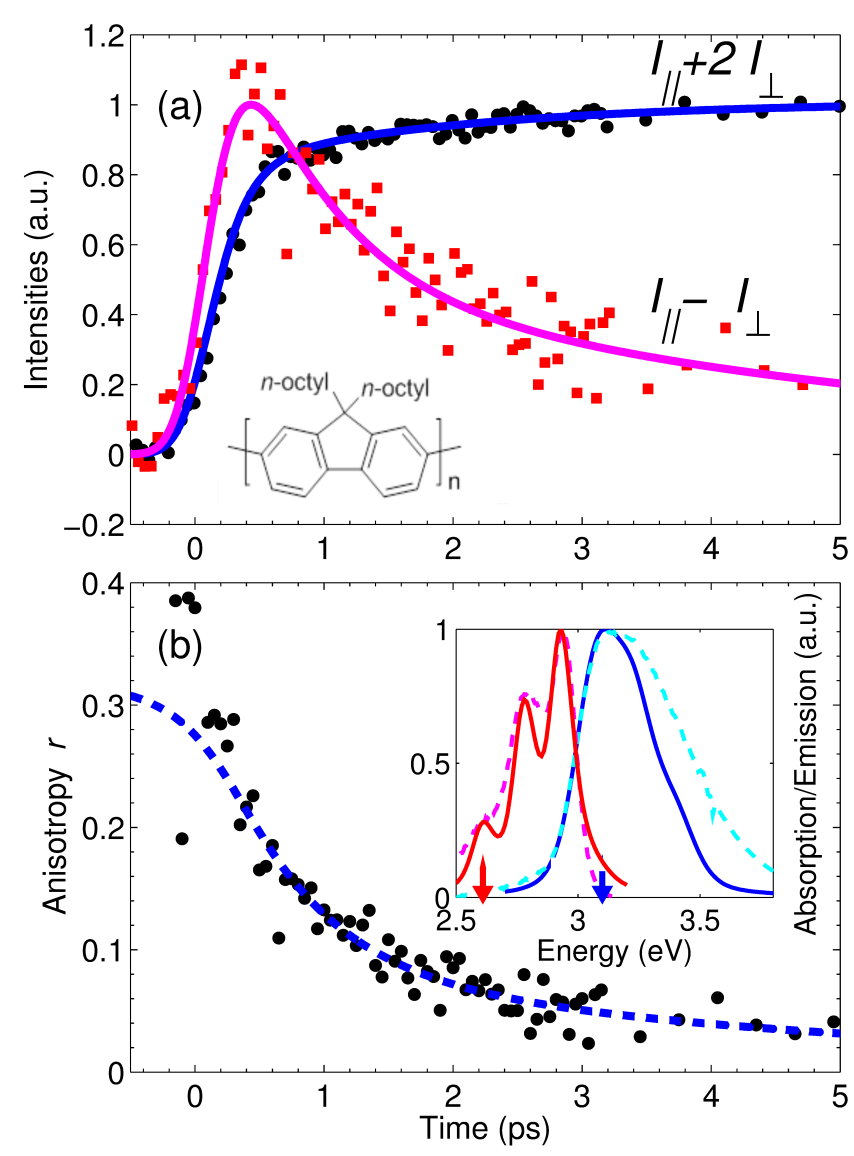

Figure 1: (a) Measured magic angle and intensity difference and (b) the resultant decay of fluorescence anisotropy for PF8 film. The PF8 chemical structure is shown as an inset to panel (a). The discrete data points are raw experimental results and lines are fitted traces including the instrument response function used to extract the reported decay times. The inset in panel (b) shows a comparison of experimental absorption and emission spectra (dotted lines) with the spectra reconstructed from the dynamical simulations. Excitation is at 400 $\mathrm{nm}$ (blue arrow) and PL detection at $475 \mathrm{~nm}$ (red arrow). We have included only one electronic transition; hence the small discrepancy between the experimental and theoretical spectra at high energies. 
the excitation laser field or any prompt higher energy emission from the film.

Using the fitting procedure described above we find that the anisotropy fits to time constants of $680 \pm 300 \mathrm{fs}$ and $4.8 \pm 2 \mathrm{ps}$ with amplitudes of 0.24 and 0.09 respectively. These values are considerably different from the fitted time constants of the magic angle dynamics and show that as well as electronic and geometric relaxation, dipole reorientation is occurring on fast and slower timescales. The instrument response does not enable us to reliably resolve the expected initial anisotropy of $0.4 ;^{48}$ instead around $t \approx 0$ and earlier times, we observe noisy anisotropy values as the total detected intensity is very low, with the fitted $r(t=0)$ of 0.33 .

\section{Discussion}

The decay of the anisotropy arises from the hopping of excitons from the chromophore where they were photo-excited to nearby chromophores oriented in a different direction. To try and understand our measurements we calculate the average Förster rate for exciton hopping in the following simple model.

The Förster hopping rate ${ }^{49,50} \gamma_{F}=1 / \tau_{F}$ between two chromophores $i$ and $j$ is : $\gamma_{F}=$ $2 \pi / \hbar \times\left|g_{i j}\right|\left|V_{i j}^{F}\right|^{2}$. In this expression $g_{i j}$ is the spectral overlap between the exciton donor and acceptor chromophores which we obtain from the measured spectral overlap between the normalized film absorption, $\mathcal{A}(\hbar \omega)$, and emission, $\mathcal{E}(\hbar \omega)$, spectra.

$$
\left|g_{i j}\right|=\frac{\int \mathcal{A}(\hbar \omega) \mathcal{E}(\hbar \omega) d(\hbar \omega)}{\int \mathcal{A}(\hbar \omega) d(\hbar \omega) \int \mathcal{E}(\hbar \omega) d(\hbar \omega)} .
$$

We calculate a spectral overlap of $\left|g_{i j}\right|=$ $0.296 / \mathrm{eV}$, from the experimental spectra presented in the inset of figure $1(\mathrm{~b}) . V_{i j}^{F}$ is the coupling energy between donor and acceptor chromophores $i$ and $j$. The most tractable approach to obtain this interaction energy is based on the line-dipole approximation ${ }^{51,52}$, which can show high accuracy at very low calculation cost, with values very close to full quantum chemistry calculations. The simpler point-dipole approximation is not valid here for chromophore separa- tions typical of films. ${ }^{52,53} V_{i j}^{F}$ is calculated using:

$$
V_{i j}^{F}=\sum_{i, j}^{N} \frac{\boldsymbol{\mu}_{A_{i}} \cdot \boldsymbol{\mu}_{B_{j}}}{\left|\boldsymbol{d}_{A_{i} B_{j}}\right|^{3}}-3 \frac{\boldsymbol{\mu}_{A_{i}} \cdot \boldsymbol{d}_{A_{i} B_{j}} \boldsymbol{\mu}_{B_{j}} \cdot \boldsymbol{d}_{A_{i} B_{j}}}{\left|\boldsymbol{d}_{A_{i} B_{j}}\right|^{5}}
$$

where $\boldsymbol{d}_{A_{i} B_{j}}$ is the vector between the centres of the sub-dipoles $\boldsymbol{\mu}_{A_{i}}$ for chromophore $A$ and $\boldsymbol{\mu}_{B_{j}}$ for chromophore $B$. In the improved line-dipole approximation, ${ }^{53}$ the sub-dipole moment $\boldsymbol{\mu}_{A_{i}}$ is given by:

$$
\boldsymbol{\mu}_{A_{i}}=\frac{\left|\sin \left(\frac{i P \pi}{N}\right)\right|}{\sum_{j}\left|\sin \left(\frac{j P \pi}{N}\right)\right|} \boldsymbol{\mu}_{A}
$$

and similarly for $\boldsymbol{\mu}_{B_{i}}$. Here we use $P=30$ and $N=50$, values found to well reproduce the quantum chemistry calculations for all possible molecular orientations. ${ }^{53}$ The transition dipole length $l$ and dipole moment $\mathbf{d}$ are obtained from quantum chemistry calculations following reference ${ }^{53}$, resulting in $l_{a b s}=5.4 \mathrm{~nm}$ and $d_{a b s}=4.65 e \AA$ in absorption and $l_{e m}=4.3 \mathrm{~nm}$ and $d_{e m}=4.74 e \AA$ in emission.

What remains is to specify the size, separation, and orientation of the molecules. Typical values for the polyfluorene conjugation length ${ }^{54}$ correspond to about eight monomer units. Based on this, we model the polyfluorene film as an ensemble of fluorene octamers. For the film under study, we measured a film density of $0.88 \pm 0.03 \mathrm{~g} / \mathrm{cm}^{3}$ for PF8 (see Supporting Information for details of this measurement), taken with the molar mass of a PF8 octamer results in an average spacing of $a=$ $1.81 \mathrm{~nm}$.

Excitation transfer between parallel molecules will lead to no change in the anisotropy, while hops between molecules which are perpendicular have zero dipole coupling such that no hopping occurs. To get a lower bound on the expected hopping times, we assume a mean angle between all chromophores of $\frac{\pi}{4}$. (Note that we have checked that doing the angle average correctly leads to only a small correction to this simplified assumption).

The resulting interaction strength for an average molecular pair then is $4.63 \mathrm{meV}$, corre- 
sponding to a Förster hopping time of $\tau_{F}=$ $16.5 \mathrm{ps}$. This time is much larger than the experimentally observed $0.68 \mathrm{ps}$ time for the fluorescence anisotropy decay. The reason for the failure of this simple estimate is that the interactions which dominate the early-time dynamics in the film are not well captured in a model based on the average properties of the film as we shall see in the next section.

\section{Theory}

We now present a predictive microscopic model of the exciton dynamics following optical excitation of a conjugated polymer film. Our model does not include coherent energy transfer processes. Indeed such processes would introduce some unknown fitting parameters; instead we want to produce a model that is not dependent on any fitting parameters. Contrary to the calculations presented in the previous discussion, this microscopic model includes the structural and spectral morphology of the film. It is here applied to polyfluorene. It is based on a microscopically founded set of rate-equations tracking the probabilities $n_{i}^{0}$ and $n_{i}^{1}$ for each chromophore $i$ to be in an excited state, either in the Franck-Condon state, just after a vertical excitation, with the chromophore being excited but still in its ground-state geometry, or in the fully-relaxed excited state. The upper indices, 0 or 1 , denote the ground state geometry, $S_{0}$, or the excited state geometry after vibrational relaxation, $S_{1}$, so that the density of probability of Franck-Condon states is $n_{i}^{0}$, and of fully relaxed excited states is $n_{i}^{1}$. We include only one electronic transition in our model, as higher electronic transitions do not play any roles at the excitation wavelengths used, do not overlap in the absorption/emission spectra and would unnecessarily complicate our model for no practical gain. The inter- and intra-molecular processes included in the model are illustrated in figure 2 in a schematic fashion. The corre-

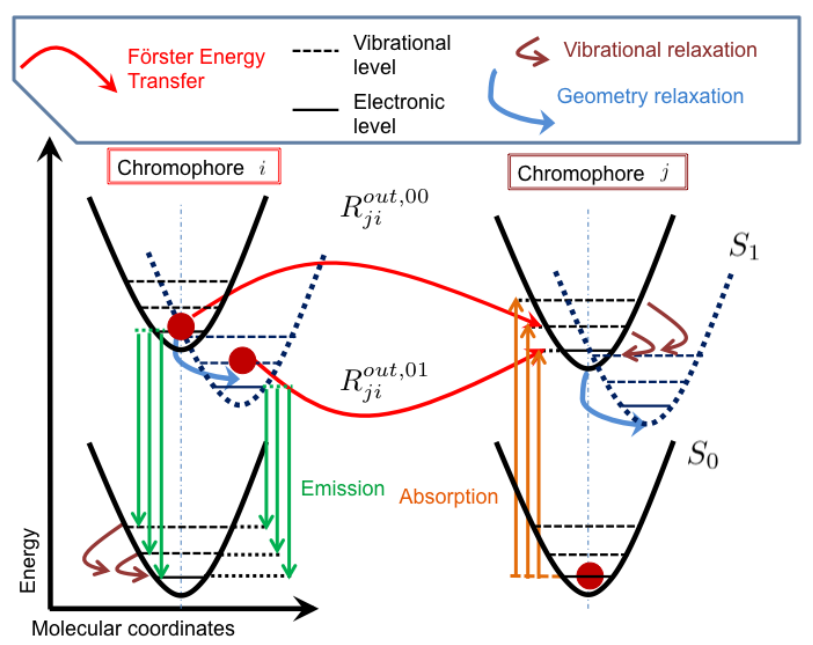

Figure 2: Schematic of the mechanisms included in the microscopic model including absorption, emission, molecular relaxation and Förster type energy transfer between chromophores $i$ and $j$.

sponding equations of motion read:

$$
\begin{aligned}
\frac{d n_{i}^{0}}{d t} & =\mathcal{I}_{i}^{\text {pump }}-\left(\gamma^{x}+\gamma^{r}+\sum_{j \neq i} R_{j i}^{\text {out }, 00}\right) n_{i}^{0} \\
& +\sum_{j \neq i}\left(R_{j i}^{\text {in, }, 01}+R_{j i}^{\text {in }, 00}\right)\left(1-n_{i}^{1}-n_{i}^{0}\right)(5) \\
\frac{d n_{i}^{1}}{d t} & =\left(\gamma^{x}-\gamma^{r}-\sum_{j \neq i} R_{j i}^{\text {out }, 01}\right) n_{i}^{1} .
\end{aligned}
$$

The first term in Eqn. (5) is the source term for pumping chromophore $i$ with dipole moment $\boldsymbol{d}_{i}$ and local field $\boldsymbol{E}$ :

$$
\mathcal{I}_{i}^{\text {pump }}=\left(1-n_{i}^{1}-n_{i}^{0}\right) \Gamma_{i}(\hbar \omega)\left|\boldsymbol{d}_{i} \cdot \widetilde{\boldsymbol{E}}(t)\right|^{2}
$$

Optical beam propagation effects are unimportant here for the thin-film dynamics. For an initially unexcited molecular ensemble, optical pumping is vertical from the ground state resulting in initially excited molecules in the $S_{0}$ geometry. ${ }^{36}$ Equation (7) is derived from the linear response of a chromophore system assuming a relatively low pumping intensity in Rotating-Wave Approximation with $\widetilde{E}(t)=$ $E(t) \mathrm{e}^{i \omega_{0} t}$ for photon energy $\hbar \omega_{0}$ (see Supporting Information for details). The spectral overlap between the chromophore absorption line 
shape $\mathcal{A}_{i}(\hbar \omega)$ (equation (12) of the Supporting Information) and the source,

$$
\Gamma_{i}(\hbar \omega) \propto \int E(\hbar \omega) \mathcal{A}_{i}(\hbar \omega) d(\hbar \omega),
$$

contains the Fourier Transform $E(\hbar \omega)$ of the optical field envelope $E(t)$. The Pauli blocking term $\left(1-n_{i}^{1}-n_{i}^{0}\right)$ in Eqn. (7) limits each chromophore to one exciton excitation only. The second term of Eqn. (5), and the first term of Eqn. (6), govern the transfer of Franck-Condon states into vibrationally-relaxed excited-states. The radiative decay of excitons is given by $\gamma^{r}=$ $1 / \tau^{r}$, with the radiative lifetime $\tau^{r}$. The remaining terms in the equations are the Försterhopping rates for hops from molecules $i$ to $j$ and vice versa. $R_{j i}^{o u t, 0 p}$ corresponds to exciton hopping from chromophore $i$ in geometry $S_{p}$ to an unexcited chromophore $j$ in its ground state geometry $S_{0} . \quad R_{j i}^{i n, 0 p}$ deals with hopping from an excited chromophore $j$ in the $S_{p}$ geometry to the chromophore $i$ in its ground-state geometry. These rates are given by:

$$
\begin{aligned}
R_{j i}^{o u t, 0 p} & =\frac{2 \pi}{\hbar}\left(1-n_{j}^{1}-n_{j}^{0}\right)\left|g_{j i}^{0 p}\right|\left|V_{j i}^{0 p}\right|^{2} \\
R_{j i}^{i n, 0 p} & =\frac{2 \pi}{\hbar} n_{j}^{p}\left|g_{i j}^{0 p}\right|\left|V_{i j}^{0 p}\right|^{2}
\end{aligned}
$$

with the Coulombic (dipole-dipole) interaction $V_{i j}^{0 p}$ between the exciton on chromophore $i$ in the ground state, and chromophore $j$ in the excited state and in the $S_{p}$ geometry. We use the improved line-dipole approximation ${ }^{53}$ to calculate the interaction matrix elements as detailed in the Supporting Information. The corresponding spectral overlap integrals $g_{i j}^{0 p}$ are obtained from the normalized emission lineshape of chromophore $j$, in the $S_{p}$ geometry, with the normalized absorption lineshape of chromophore $i$. The spectral overlaps are calculated separately for each pair of chromophores in the film with the spectra determined as detailed below. To include inhomogeneous broadening, each chromophore possesses its own absorption transition energies. These energies are randomly generated with a Gaussian distribution of width $\sigma$ (the inhomogeneous broadening parameter), centered around the electronic ab- sorption energy, $E_{i}^{a b s}$. For each chromophore, the emission energy is correlated with its absorption energy.

On top of the electronic transitions, a vibrational substructure is included, which was found to be essential in an earlier study. ${ }^{50}$ The spectrum of a single chromophore is modelled as a sum of Lorentzian lines that are weighted to reproduce the observed vibrational progression. Each Lorentzian has a width $\gamma$ referred to as the homogeneous broadening. The computed absorption spectrum of the film can then be obtained by integration over the vibronic peaks and all chromophores and orientation of the respective absorption dipoles:

$$
\mathcal{A}(\hbar \omega)=\hbar \omega \sum_{i} \mathcal{A}_{i}
$$

with

$\mathcal{A}_{i}=L\left(E_{i}^{a b s}, \hbar \omega\right)+\sum_{k} \alpha_{k}^{v i b} L\left(E_{i}^{a b s}+\delta E_{k}^{v i b}, \hbar \omega\right)$

$\alpha_{k}^{v i b}$ and $\delta E_{k}^{v i b}$ denote the strength and energetic offset of the $k$-th vibrational level, respectively, and $L(x, \hbar \omega)$ is a normalized Lorentzian centred at $x$ with width $\gamma$. The emission spectrum depends on the time $t$ of emission as determined by the exciton dynamics:

$$
\mathcal{E}(\hbar \omega, t)=\hbar \omega \sum_{i} I_{i}(\hbar \omega, t) .
$$

Here $I_{i}$ is the time- and energy-dependent intensity emitted by each chromophore $i$ :

$I_{i}(\hbar \omega, t)=n_{i}^{1}(t) \mathcal{E}_{i}\left(E_{i}^{e m}, \hbar \omega\right)+n_{i}^{0}(t) \mathcal{E}_{i}\left(E_{i}^{a b s}, \hbar \omega\right)$

with the chromophore emission spectrum

$\mathcal{E}_{i}(x, \hbar \omega)=L(x, \hbar \omega)+\sum_{k} \alpha_{k}^{v i b} L\left(x-\delta E_{k}^{v i b}, \hbar \omega\right)$.

In order to model a given film with the theoretical approach outlined above, a number of physical parameters need to be specified. Knowledge of the transition dipoles is required both for optical excitation and de-excitation 
and for the Förster type inter-molecular coupling. Further required is the morphology of the sample (position and orientation of each transition dipole), and the spectral properties, both globally for the ensemble as well as for individual chromophores. Here we extract all the parameters needed either from independent experimental measurements or from theoretical quantum chemistry calculations for individual chromophores and pairs of chromophores. The films are assumed to be spin coated with a preferential orientation of the molecular chains in the plane, the film density was measured, and a typical average chromophore conjugation length of 8 fluorene repeat units was used for the polyfluorene film. ${ }^{53,54}$ The homogeneous and inhomogeneous broadenings of optical transitions was determined by reproducing the measured absorption and emission line shapes of the film. We found that only one combination of the two parameters consistently reproduced both absorption and emission simultaneously. Full details on the parameters are given in table 1 and in the Supporting Information.

Once all the parameters are fixed, the set of coupled equations of motion, equations (5) and (6), can be solved for a given excitation situation (defined by intensity, frequency, polarization, and temporal shape of the light pulse) and a given film (defined by the properties of the individual chromophores, sample morphology, and spectral properties). In this work, this typically means solving the relevant set of coupled equations of motion for a molecular ensemble of about 125000 chromophores. From this solution, physical quantities such as time-resolved polarization anisotropy of the emitted fluorescence or spectral and spatial diffusion can be extracted from the model and directly compared with experiment as will be discussed in detail. Below we further demonstrate the capabilities of our a microscopic theoretical approach to provide insightful information on quantities not directly accessible in experiment.

We neglect coherent contributions to the dynamics as these typically only significantly contribute on very short timescales. Furthermore, below we demonstrate very good agreement with the de-polarization dynamics observed ex-

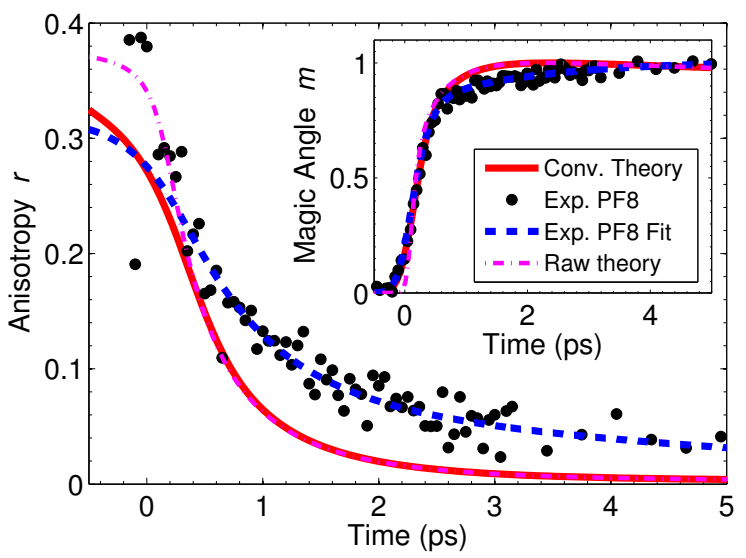

Figure 3: Comparison of the predicted fluorescence anisotropy and magic angle dynamics (inset) with the dynamics observed in experiment (the discrete data points are raw results). The "Conv. theory" result corresponds to the theory result convoluted with the instrument response function. The "Raw theory" results are unconvoluted.

perimentally, indicating that coherences probably only play a secondary role here. The anisotropy experiments in this section are performed at low pump intensity, therefore at this point we need not consider exciton-exciton annihilation in the computed dynamics.

Details such as the instrument response function and spectral filtering are included in the calculations. Full details on parameters and sample morphology and evaluation of results are provided in the Supporting Information; a summary of their values is presented in table 1 .

\section{Comparison of theory and experiment}

As one of the main results of the present work, figure 3 shows the computed decay of fluorescence anisotropy in comparison with the experimental data. Good agreement with experiment is found. In particular we re-iterate here that our theoretical model is purely predictive and is not a fit: no fitting procedure was used in the but all the system parameters needed were either inferred from independent experimental results or from quantum-chemical calculations. 
Table 1: Summary or all the parameters used in our model, and how they are fixed. Some of the geometrical parameters are estimated, but it has been checked that within reason different values do not alter the fluorescence anisotropy and magic angle results (see Supporting Information).

\begin{tabular}{|c|c|c|c|}
\hline \multicolumn{2}{|l|}{ Parameter } & Value & From \\
\hline \multirow{4}{*}{ Geometrical } & Conjugation length & O8 chromophore & Literature $^{54}$ \\
\hline & Oligomer length & $6.64 \mathrm{~nm}$ & Quantum Chemistry \\
\hline & Film density & $d=0.88 \pm 0.03 \mathrm{~g} / \mathrm{cm}^{3}$ & Experimental measure \\
\hline & Closest approach distance & $0.3 \mathrm{~nm}$ & Estimate \\
\hline \multirow{7}{*}{ Spectral } & Homogeneous broadening $\gamma$ & $33 \mathrm{meV}$ & \multirow{3}{*}{$\begin{array}{l}\text { Fit to experimental spectra; } \\
\text { see Supporting Information } \\
\text { for error discussion. }\end{array}$} \\
\hline & $\begin{array}{l}\text { Absorption inhomogeneous } \\
\text { broadening } \sigma\end{array}$ & $70 \mathrm{meV}$ & \\
\hline & Absorption energy peak & $3.07 \mathrm{eV}$ & \\
\hline & Emission energies & $E_{i}^{e m}=0.48 E_{i}^{a b s}+1.50$ & $\begin{array}{l}\text { Fit to experimental spectra; } \\
\text { Quantum Chemistry }\end{array}$ \\
\hline & Transition dipole moments & $d_{a b s}=4.65 \mathrm{eA}$ and $d_{e m}=4.74 \mathrm{eA}$ & \multirow{2}{*}{ Quantum Chemistry } \\
\hline & Transition dipole lengths & $l_{a b s}=5.4 \mathrm{~nm}$ and $l_{e m}=4.3 \mathrm{~nm}$ & \\
\hline & $\begin{array}{l}\text { Molecular geometry } \\
\text { relaxation time } \tau^{x}\end{array}$ & $100 \pm 50 \mathrm{fs}$ & Literature $^{1}$ \\
\hline
\end{tabular}

In figure 3 we find, that both a fast subpicosecond initial decay and the slower decay on longer times are reproduced by the simulations. These results demonstrate the capabilities of our microscopically-founded model. In contrast to the simple average estimate above we obtain good agreement with experiment, indicating that indeed the depolarisation dynamics is determined by Förster-type energy transfer between individual, interacting molecules with properties that can not easily be traced back to the average properties of the film. This result also indicates that solely incoherent Förster energy transfer can give rise to the sub-ps anisotropy decay observed experimentally. No other mechanisms, such as coherent energy transfer, need to be invoked to explain this ultra-fast decay.

With further, to some extent arbitrary, adjustment of selected model parameters, without doubt an improved agreement with experiment could be possible. However, we chose not to do this, as this would undermine the predictive character of our microscopic approach and not provide any deeper understanding of the underlying physics. In modelling the inter-molecular interaction matrix elements we do not include any background dielectric constant. For nearby neighbours this assumption is fully justified. For neighbours further apart from each other, one may argue that a distance-dependent dielectric function should be included. This would slow down the computed dynamics slightly and further improve the agreement with experiment.

Note that the anisotropy, both experimental and theoretical, do not start at $r=0.4$, the value to be expected for excitation of a random rod distribution. The experimental instrument response is too slow to obtain an accurate measurement of the initial fast anisotropy decay. However, the "raw" theory results from the microscopic rate equations (without convolution with the instrument response function) obviously resolve this decay and an initial value of $r=0.4$ is observed (cf. figure 3 ).

\section{Spectral diffusion and dynamical exciton localization}

In our microscopic model we explicitly compute the spatio-temporal excitation dynamics for a large and inhomogeneously broadened ensemble of chromophores in the film. This allows 


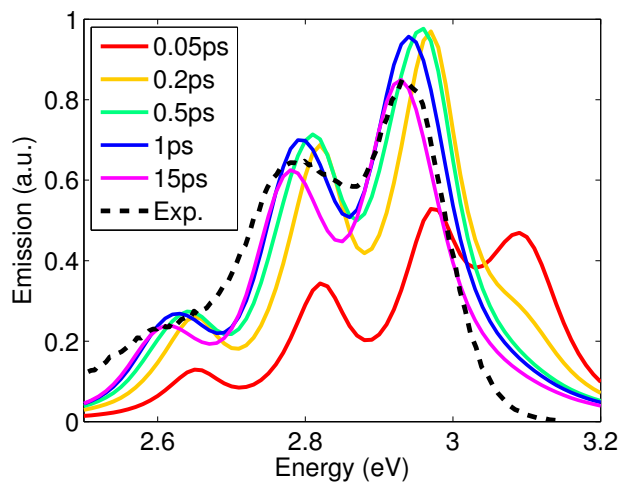

Figure 4: Simulated time-resolved emission spectra (solid lines), compared with the experimental emission spectrum at 10 ps (dashed line). The high energy feature at early times stems from molecules emitting before vibrational relaxation occurs.
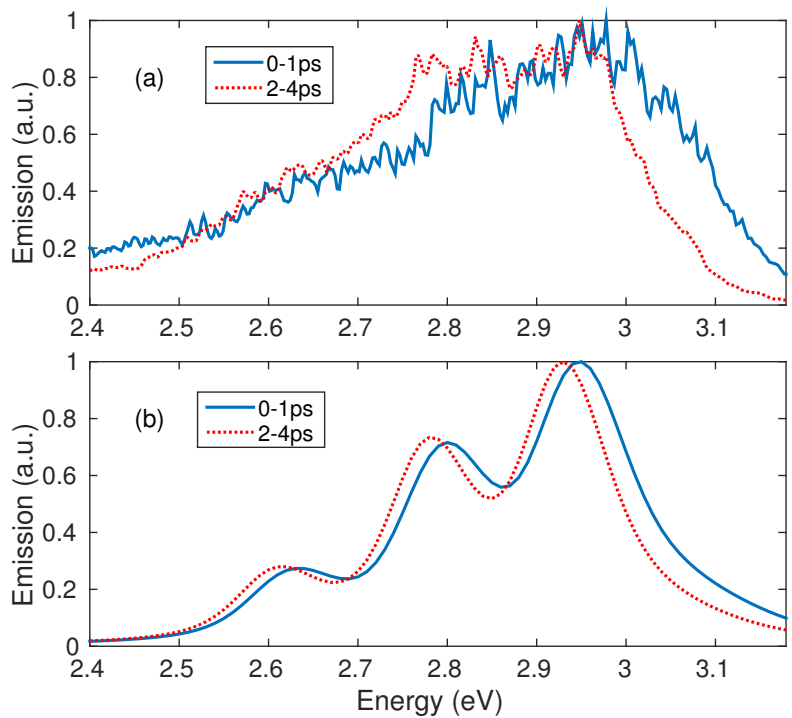

Figure 5: (a) Experimental and (b) theoretical spectral energy shift over time, integrated from either 0 to 1 ps (blue-solid) or 2 to 4 ps (reddotted). us to gain additional insight into phenomena that accompany fluorescence de-polarization as discussed in the previous section but are more difficult (and only indirect) to access experimentally and not accessible in a globally averaged model. Figure 4 shows the simulated time-resolved emission spectrum as it evolves after photoexcitation. With these theoretical results, we observe a very fast (a few hundreds of femtoseconds) shift of the emission peak to lower energies. A steady state emission is reached after approximately two picoseconds. This is consistent with the time-scales obtained for the anisotropy decay. The high energy emission peaks at very early times correspond to emission from Franck-Condon states, which are excited chromophores in the $S_{0}$ geometry, not yet vibrationally relaxed. Such unrelaxed chromophores have a strong spectral overlap with the absorption of unexcited chromophores which is responsible for efficient inter-chromophore excitation transfer at early times. In figure 5, we compared the experimentally recorded time-resolved emission spectra with the results of the calculations. A fast redshift of the 0-0 peak is evident in both experiment and simulation. Overall the agreement with the time-resolved spectral experiments is very good. For a more detailed picture, figure 6 shows the simulated exciton occupation probability resolved over the respective chromophore emission energies. We clearly see that at the earliest times, the pumping process excites molecules at well defined energies, with a shape roughly resembling the spectral profile of the pump. This is followed by the subsequent migration of these initial excitations to molecules lower in energy, where the exciton probability gets more and more localised also in space. Figure 7 illustrates the spatio-temporal relaxation to low energy sites further. Shown is a $3 \mathrm{D}$ plot of the spatially resolved excitation probability inside the sample at different points in time. The chromophore excitation energy is encoded in the color. We find that the chromophores initially excited are numerous, quite close to each other and spread all over the sample. As the excitations get trapped at lower energy chromophores, these excited chromophores 
are on average further apart from each other, which together with the spectral diffusion observed gives rise to much slower inter-molecular excitation transfer.
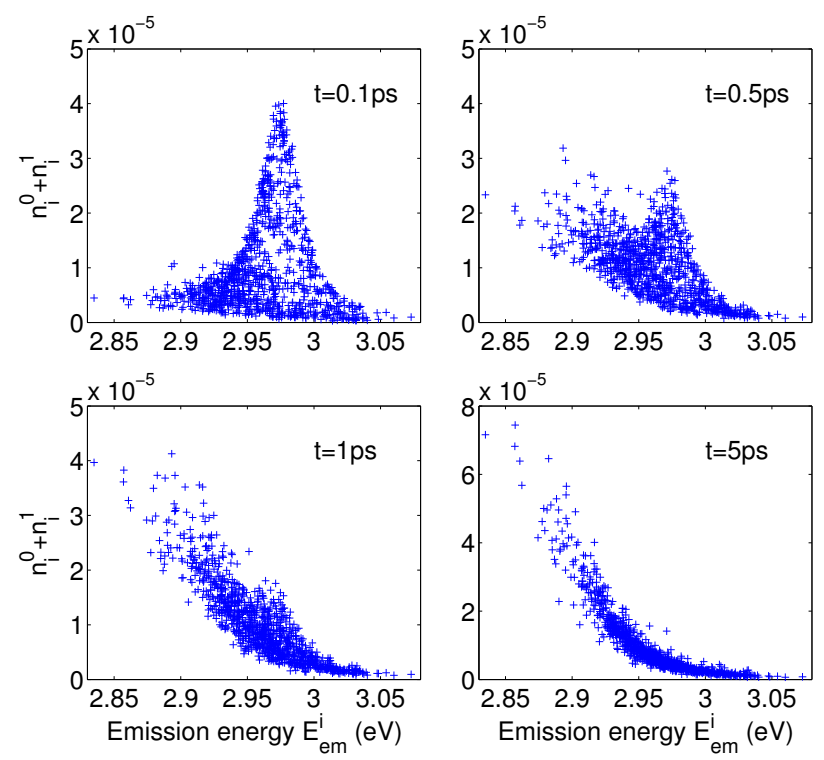

Figure 6: Total exciton occupation probability as a function of the chromophore emission energy. Note the change of scale on the $t=5 \mathrm{ps}$ plot and that for clarity only 1000 of the 125, 000 modelled chromophores are shown.

Going more into the details of our results, we have calculated that the average exciton energy decreases from $3.07 \mathrm{eV}$ to $2.95 \mathrm{eV}$ in about 1 ps. After 5 ps the exciton energy standard deviation stays almost constant, showing that no more net exciton dynamics take place from this time onwards and the system has reached a dynamical equilibrium state. In addition to the ever increasing average distance of excited chromophores with time, the microscopic dynamics is further slowed down by the increasing excitation probability of the individual lowenergy sites leading to increased blocking of the inter-chromophore exciton transfer. In detail, there is still a very slow increase of the standard deviation, because the excitons will try to reach even lower energy chromophores. However, these chromophores are not numerous and therefore very widely spread across the sample, making it difficult for the excitons to diffuse to them, especially as these low energy chromophores will already have a high exciton prob- ability and therefore experience Pauli blocking.

It is possible to analyze the Förster-type interaction rates involved in the model in detail, to deduce the number and the strength of these interactions, and which chromophores are primarily involved. We have calculated that about $11 \%$ of chromophores possess an interaction term $R_{j i}^{\text {out }, 01}$ with another chromophore leading to dynamics faster than $1 \mathrm{ps}$, and $30 \%$ between 1 and $2 \mathrm{ps}$, so that more than $40 \%$ of the molecules have at least one "neighbor" for which the interaction is faster than $2 \mathrm{ps}$, explaining the fast anisotropy decay observed. We find that the fastest interactions correspond to chromophores with the largest energy difference, consistent with the spectral overlap argument given above. This shows that we should indeed expect the fast exciton hopping to be over after a few picoseconds. Furthermore, most excitons will directly go to the low energy sites, without going through molecules of intermediate energies. In the results shown, with our excitation energy at $3.10 \mathrm{eV}$, we initially excite the interacting pairs with the strongest interactions, explaining the fast depolarisation. When pumped at lower energies, the de-polarization dynamics slows down somewhat as shown in the Supporting Information. We can also extract the separations of the chromophore pairs which lead to the fastest decay rates. At just over $3 \mathrm{~nm}$, the center-to-center chromophore separations of the fastest Förster rates are relatively large; significantly larger than the average chromophore separation in the plane of $0.9 \mathrm{~nm}$. Therefore, we find that the fastest excitation transfer does not occur between an excited chromophore and its nearest neighbor, but between an excited chromophore and a spectrally matched lower energy chromophore somewhere in the vicinity. On this note we would like to mention that we find the exciton dynamics not to be very sensitive to geometric parameters used for the generation of the chromophore ensemble. Rather than the distance of the closest neighbors, the spatial parameter of importance is the film density. We have explicitly checked different packings of the chromophores to build the film as well as different average chromophore lengths. and find the re- 

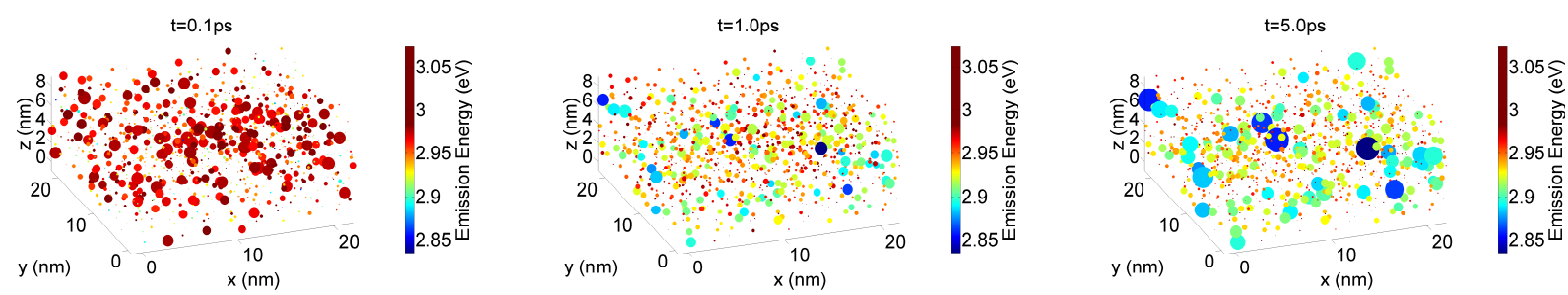

Figure 7: Three-dimensional visualization of a small portion of the simulated film. Molecular excitations are represented by spheres centred at the respective chromophore position. Each sphere's surface area indicates the chromophore's exciton density and the emission energy is encoded in the color. The plots at $t=0.1 \mathrm{ps}, t=1 \mathrm{ps}$ and $t=5 \mathrm{ps}$ show the system's excitation shortly after the peak of the pump intensity at $\mathrm{t}=0$. Both spatial localization at low-energy sites and spectral diffusion are clearly evident.

sults are virtually identical with the results presented here (see Supporting Information for additional data).

\section{Exciton diffusion}
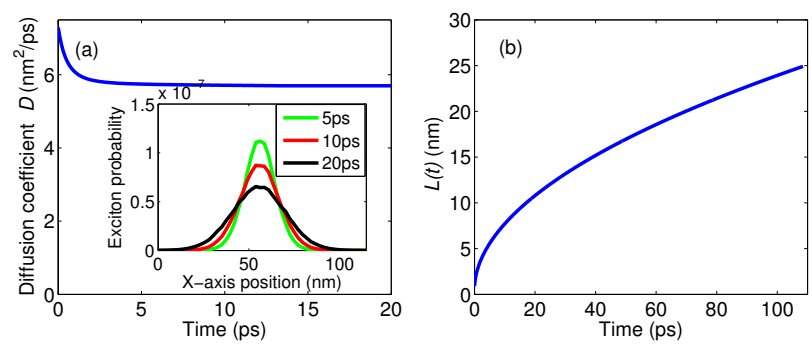

Figure 8: Simulated exciton diffusion coefficient $D$ in (a) and calculated diffusion length (equation 18) in (b) for excitation of the sample with a Gaussian pump profile along the $x$ axis. The inset in (a) shows the total exciton probability as a function of the $x$ coordinate, at different times following excitation.

The exciton diffusion length $L_{D}$ is the average distance travelled by excitons during their lifetime. At low excitation density, detailed information on exciton diffusion can be obtained from our hopping model. To this end, in our simulations we excite the film with a pump with a Gaussian spatial profile. An example of this excitation scenario is provided in the inset of figure 8(a). To characterise the spatial and temporal variation of the hopping we can make a comparison with the standard equation for dif- fusion in one dimension, ${ }^{55}$

$$
\frac{\partial n}{\partial t}=D(t) \frac{\partial^{2} n}{\partial x^{2}}
$$

With an initial gaussian distribution of standard deviation $\sigma_{D}^{0}$, the standard deviation $\sigma_{D}(t)$ of the resulting excitation profile can be related to the diffusion coefficient ${ }^{56}$ using

$$
D(t)=\frac{1}{2} \frac{d}{d t} \sigma_{D}^{2}
$$

From this, we calculate the time dependent diffusion distance $L(t)$,

$$
L^{2}(t)=\int_{0}^{t} D\left(t^{\prime}\right) d t^{\prime}=\frac{1}{2}\left(\sigma_{D}^{2}(t)-{\sigma_{D}^{0}}^{2}\right),
$$

and the conventional exciton diffusion length $L_{D}=L\left(\tau^{r}\right)$. The computed exciton diffusion coefficient $D(t)$ and the quantity $L(t)$ are plotted in figure 8. We observe two stages in the time-dependent diffusion coefficient: during the first three picoseconds, it decreases from $D \approx 7.3 \mathrm{~nm}^{2} / \mathrm{ps}$ to $D=5.7 \mathrm{~nm}^{2} / \mathrm{ps}$, and then stays approximately constant during the rest of the time computed (20 ps). The initial decrease reflects the localisation of the excitons from the high energy sites where they were created to the low energy sites, and is consistent with the timescale observed with the fluorescence anisotropy decay. In this initial three picoseconds the diffusion distance is around $3 \mathrm{~nm}$ consistent with the separation of the fastest interacting chromophores mentioned above. The diffusion mechanism after this localisation is the 
exciton transfer to other low energy sites. We assume that this is a steady-state mechanism, so that $D(t>20 \mathrm{ps})=D(20 \mathrm{ps})$. The steady state value of exciton diffusivity from our model is about four times higher than the value of $D=1.2 \mathrm{~nm}^{2} / \mathrm{ps}$ obtained from exciton-exciton annihilation experiments assuming an annihilation radius of $1 \mathrm{~nm} .{ }^{23}$ Similar diffusivity value of $1.44 \mathrm{~nm}^{2} / \mathrm{ps}$ has been obtained in glassy films of another polyfluorene derivative by measuring energy transfer to dispersed molecules of tetraphenyl porphyrin ${ }^{57}$. At $L_{D}=L\left(\tau^{r}=\right.$ $100 \mathrm{ps}$ ), we calculate an exciton diffusion length of approximately $24 \mathrm{~nm}$. Using the diffusivity and the observed 114 ps PL lifetime observed by Shaw et $a^{23}$ implies a diffusion length of $12 \mathrm{~nm}$. As all parameters in our model are obtained from quantum chemistry, matching to optical spectra or other measurements with no adjustment; we judge such agreement to be satisfactory.

\section{Exciton-exciton annihilation at el- evated pump intensities}

At elevated excitation densities, Auger-type bi-molecular exciton-exciton annihilation processes play a significant role in the excitation dynamics. Exciton-exciton annihilation manifests itself for example in the dynamics of the emitted photoluminescence after photoexcitation of the molecular film. Experimental results for polyfluorene of different types of morphology were reported previously. ${ }^{23}$ Here we extend our microscopic model detailed above to include exciton-exciton annihilation events in the dynamics. To this end, we add the term

$$
\gamma_{i}^{X X}=-\sum_{j \neq i} R_{j i}^{X X} n_{j}^{1}
$$

inside the bracket of equation (6). ${ }^{58}$ The physical process leading to exciton-exciton annihilation is quadratic in the excitation density and correspondingly only contributes at sufficiently high excitation densities. The quantity

$$
R_{j i}^{X X}=\frac{2 \pi}{\hbar}\left|g_{j i}^{X X}\right|\left|V_{j i}^{X X}\right|^{2}
$$

includes both the spectral overlap $g_{j i}^{X X}$ of chromophores $i$ and $j$ contributing to the annihilation as well as the relevant dipole-dipole interaction matrix element $V_{j i}^{X X}$. In accordance with the underlying Auger-type scattering mechanism, the annihilation involves the transition dipoles and spectral densities of molecule $i$ between ground and first excited state and the excited-state absorption of molecule $j$ between first excited state and higher-lying excited states. These spectral overlaps and interaction matrix elements are calculated in the same way as detailed for the Förster rates above, but with the excited-state absorption properties instead of the regular absorption for one of the molecules. At moderate densities and above the geometry relaxation in the excited state is much faster than the annihilation so that both molecules are in their relaxed $S_{1}$ geometries. The relaxation of the double excitation on molecule $j$ back to the lowest excited state is taken to be instantaneous on the timescales of interest.

Quantitative information on the excited-state absorption strength near the chromophore's fundamental excitation energy is at present lacking. In this spectral range, the ESA overlaps with the strong one-photon absorption, making accurate measurements difficult. To include the bi-molecular annihilation we make the following plausible assumptions. The ESA dipole is oriented in the same spatial direction as the regular absorption dipole, ${ }^{59}$ for the dipole length we assume an average value of $l_{X X}=5 \mathrm{~nm}$, the ESA homogeneous line width we assume twice as large as for the regular absorption, $\gamma_{X X}=2 \gamma$, and we take the ESA absorption at twice the emission energy of a given chromophore. The latter assumption is consistent with the large annihilation rates previously reported for PF8 films. ${ }^{23}$ The one remaining parameter, the effective ESA absorption dipole $d_{X X}$ magnitude, we fix against the experiment at a given excitation power as detailed below.

An exciton-exciton annihilation rate $\eta^{X X}(t)$ can be formally defined on a macroscopic level 
through the following equation: ${ }^{23}$

$$
\frac{d n}{d t}=-\frac{n(t)}{\tau^{r}}-\eta^{X X} n(t)^{2}
$$

Here $n(t)$ is the total exciton density inside the sample and $\tau^{r}$ is the radiative decay time. In reference ${ }^{23} \eta^{X X}(t)$ was determined from experiment for polyfluorene films. There it was found that $\eta^{X X}$ only varies in time within the first $15 \mathrm{ps}$ and then becomes constant at $\eta^{X X} \approx$ $1.5 \times 10^{-8} \mathrm{~cm}^{3} / \mathrm{s}$. For a given excitation density, we can also extract $\eta^{X X}(t)$ from our microscopic model, where the total excitation density is given as $n(t)=\sum_{i}\left(n_{i}^{1}(t)+n_{i}^{0}(t)\right) / V$, where $V$ is the sample volume $\left(V=a^{2} \times b\right)$ In the calculations, we find that $\eta^{X X}$ is basically independent of the pump intensity (see figure 9). To reproduce the correct long-time value of the annihilation rate $\eta^{X X}(t)$, we adjusted the excitedstate absorption dipole to $d_{X X}=2.51 \mathrm{e} \AA$, i.e. somewhat weaker than the one-photon transition.

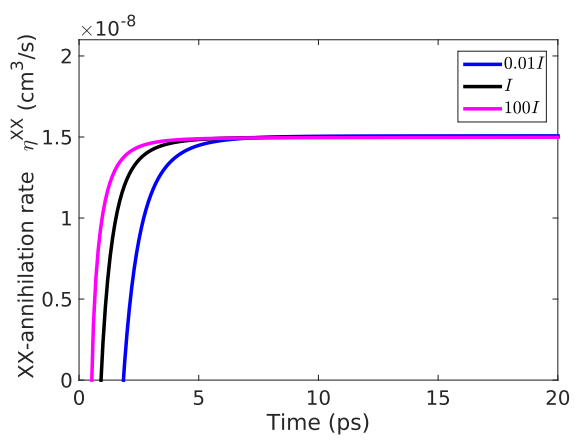

Figure 9: Simulated exciton-exciton annihilation rates $\eta^{X X}$ as a function of time, for three different pump intensities, labelled as in figure 10 - the strongest pump leads to significant exciton-exciton annihilation, whereas the weakest pump barely affects the decay of the total exciton density.

Furthermore Shaw et al. ${ }^{23}$ measured the temporal decay of the photoluminescence for a range of different pump intensities. With all the parameters fixed above, in figure 10 we compare our computed data with these measurements. All the intensities are given in units of the intensity $I$ best reproducing the PL decay at the lowest excitation power shown. As clearly visible in figure 10, excellent agreement of the computed and experimental data is found for a wide range of intensities. We would like to note that the decay is not a simple exponential decay but can be fitted with a three-exponential fit as detailed in reference ${ }^{23}$. To underline the agreement further, it is instructive to also extract the effective exciton lifetimes $\tau_{\text {eff }}^{r}$ from the data shown and compare with the experiments. The effective exciton lifetime is the times it takes to reach 1 /e of the peak total exciton density. The result is shown in figure 11 and again excellent agreement is found. We also note that if the exciton migration was artificially switched off in the calculations, (by setting hopping matrix elements to zero) we could not achieve a satisfactory agreement of the computed results with the experiments. This emphasises the important interplay of migration/diffusion and annihilation on a microscopic level. As this interplay is included in the model, we can also extract an effective density dependent diffusion length $L\left(\tau_{\text {eff }}^{r}\right)$, which is also shown in figure 11 . It is clearly visible that for increasing pump intensities, the bi-molecular annihilation is the factor limiting the diffusion of excitons.

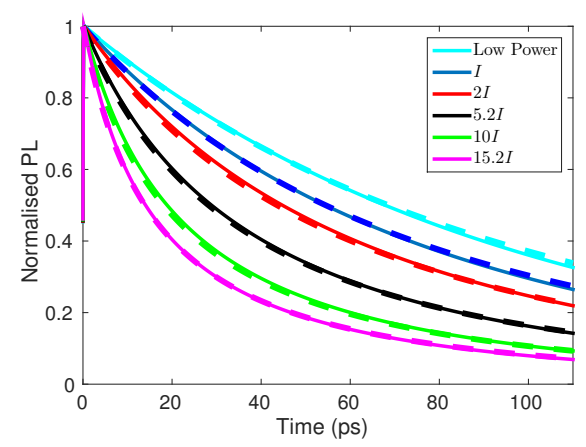

Figure 10: Photoluminescence decay for different pump intensities. Theoretical data are represented by solid lines, fits to the experimental data $^{23}$ by dashed lines.

\section{Conclusions \& Outlook}

In conclusion, we have examined the ultrafast photoluminescence depolarisation and exciton dynamics in thin-films of polyfluorene with experimental and theoretical treatments. Our finding is that the experimentally observed 


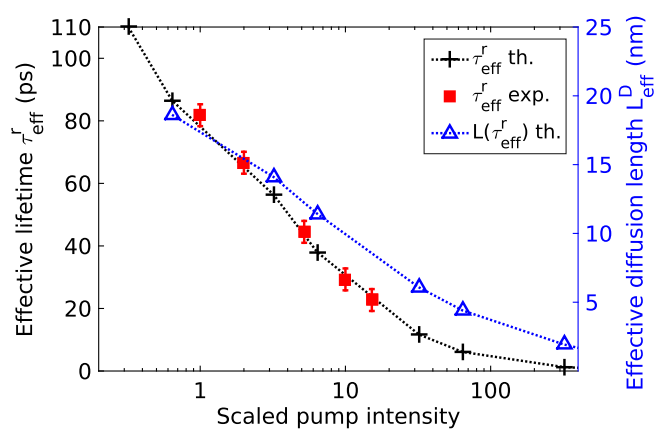

Figure 11: Variation of the effective exciton lifetime with the scaled pump intensity, as in figure 10. The theoretical data are shown as black symbols and the experimental data ${ }^{23}$ as red squares. The resulting exciton diffusion length extracted from the model is shown in blue. Lines are only a guide-to-the-eye. Error bars are shown on the experimental data.

$680 \pm 300$ fs decay of PL anisotropy - representative of electronic energy transfer - can be well described in an independently built microscopic theoretical model of the system that uses only line-dipole resonant energy transfer between the chromophores. We do not need to invoke more complex mechanisms involving EET in the strong or intermediate coupling limit in order for the model to adequately agree with the experimental results, giving good evidence that conventional line-dipole resonant energy transfer is appropriate to describe EET in thin films of polyfluorene. Beyond this conclusion, we were able to computed spectral diffusion and dynamical exciton localization using the model to give further insight into the ultrafast excited state after photoexcitation in polyfluorene. The theoretical model comprises of 125000 chromophores that are located appropriately for a spin coated thin film of the material, giving suitable spatial and energetic disorder to enable such conclusions to be drawn. Finally, at elevated excitation intensities, bi-molecular exciton annihilation was investigated and the interplay between exciton migration and annihilation was found crucial to reproduce the experimental data over a range of different intensities.

Acknowledgement We acknowledge finan- cial support from the UK EPSRC (grants EP/E065066/1, EP/E062636/1,EP/J009318/1 and EP/J009019/1), from the EPSRC Scottish Centre for Doctoral training in Condensed Matter Physics and from the European Union Seventh Framework Programme under grant agreement 321305.

Supporting Information Available: We have added details about the experimental methodology and data fitting, and about the choice and effect of parameters used in our simulations. This material is available free of charge via the Internet at http://pubs.acs . org/.

\section{References}

(1) Hwang, I.; Scholes, G. D. Electronic Energy Transfer and Quantum-Coherence in -Conjugated Polymers. Chem. Mater. 2011, 23, 610-620.

(2) Li, G.; Zhu, R.; Yang, Y. Polymer Solar Cells. Nat. Photon. 2012, 6, 153-161.

(3) Banerji, N. Sub-picosecond Delocalization in the Excited State of Conjugated Homopolymers and Donor-Acceptor Copolymers. J. Mater. Chem. C 2013, 1, 30523066 .

(4) Rivnay, J.; Mannsfeld, S. C. B.; Miller, C. E.; Salleo, A.; Toney, M. F. Quantitative Determination of Organic Semiconductor Microstructure from the Molecular to Device Scale. Chem. Rev. (Washington, DC, U.S.) 2012, 112, 5488-5519.

(5) Koch, F. P. V.; Rivnay, J.; Foster, S.; Müller, C.; Downing, J. M.; BuchacaDomingo, E.; Westacott, P.; Yu, L.; Yuan, M.; Baklar, M. et al. The Impact of Molecular Weight on Microstructure and Charge Transport in Semicrystalline Polymer Semiconductors-poly(3hexylthiophene), a Model Study. Prog. Poly. Sci. 2013, 38, 1978-1989. 
(6) Schwartz, B.; Nguyen, T.; Wu, J.; Tolbert, S. Interchain and Intrachain Exciton Transport in Conjugated Polymers: Ultrafast Studies of Energy Migration in Aligned MEH-PPV/mesoporous Silica Composites. Synth. Met. 2001, 116, 3540.

(7) Ruseckas, A.; Theander, M.; Valkunas, L.; Andersson, M.; Inganäs, O.; Sundström, V. Energy Transfer in a Conjugated Polymer with Reduced Inter-chain Coupling. J. Lumin. 1998, 76-77\%, 474477.

(8) Beljonne, D.; Pourtois, G.; Silva, C.; Hennebicq, E.; Herz, L. M.; Friend, R. H.; Scholes, G. D.; Setayesh, S.; Müllen, K.; Brédas, J. L. Interchain vs. Intrachain Energy Transfer in Acceptor-capped Conjugated Polymers. Proc. Natl. Acad. Sci. U.S.A. 2002, 99, 10982-10987.

(9) Shekhar, S.; Aharon, E.; Tian, N.; Galbrecht, F.; Scherf, U.; Holder, E.; Frey, G. L. Decoupling 2D Inter- and Intrachain Energy Transfer in Conjugated Polymers. Chem. Phys. Chem. 2009, 10, 576-581.

(10) Hennebicq, E.; Pourtois, G.; Scholes, G. D.; Herz, L. M.; Russell, D. M.; Silva, C.; Setayesh, S.; Grimsdale, A. C.; Müllen, K.; Brédas, J.-L. et al. Exciton Migration in Rigid-Rod Conjugated Polymers: An Improved Förster Model. J. Am. Chem. Soc. 2005, 127, 4744-4762.

(11) Dubin, F.; Melet, R.; Barisien, T.; Grousson, R.; Legrand, L.; Schott, M.; Voliotis, V. Macroscopic Coherence of a Single Exciton State in an Organic Quantum Wire. Nat. Phys. 2006, 2, 32-35.

(12) Ruseckas, A.; Wood, P.; Samuel, I. D. W.; Webster, G. R.; Mitchell, W. J.; Burn, P. L.; Sundström, V. Ultrafast Depolarization of the Fluorescence in a Conjugated Polymer. Phys. Rev. B 2005, 72, 115214.
(13) Collini, E.; Scholes, G. D. Coherent Intrachain Energy Migration in a Conjugated Polymer at Room Temperature. Science (Washington, DC, U.S.) 2009, 323, 369373.

(14) Hill, J.; Heriot, S.; Worsfold, O.; Richardson, T.; Fox, A.; Bradley, D. Dynamics of Förster Transfer in Polyfluorene-based Polymer Blends and Langmuir-Blodgett Nanostructures. Synth. Met. 2003, 139, 787-790.

(15) Buckley, A.; Rahn, M.; Hill, J.; Cabanillas-Gonzalez, J.; Fox, A.; Bradley, D. Energy Transfer Dynamics in Polyfluorene-based Polymer Blends. Chem. Phys. Lett. 2001, 339, 331-336.

(16) Dykstra, T. E.; Hennebicq, E.; Beljonne, D.; Gierschner, J.; Claudio, G.; Bittner, E. R.; Knoester, J.; Scholes, G. D. Conformational Disorder and Ultrafast Exciton Relaxation in PPV-family Conjugated Polymers. $J$. Phys. Chem. B 2009, 113, 656-667.

(17) Tozer, O. R.; Barford, W. Exciton Dynamics in Disordered Poly $(\mathrm{p}$ phenylenevinylene). 1. Ultrafast Interconversion and Dynamical Localization. J. Phys. Chem. A 2012, 116, 10310-10318.

(18) Binder, R.; Wahl, J.; Romer, S.; Burghardt, I. Coherent Exciton Transport Driven by Torsional Dynamics: a Quantum Dynamical Study of Phenylenevinylene Type Conjugated Systems. Faraday Discuss. 2013, 163, 205-222.

(19) Xia, R.; Stavrinou, P. N.; Bradley, D. D. C.; Kim, Y. Efficient Optical Gain Media Comprising Binary Blends of Poly(3-hexylthiophene) and $\operatorname{Poly}(9,9-$ dioctylfluorene-co-benzothiadiazole). $J$. Appl. Phys. 2012, 111, 123107-123115.

(20) Park Sung, H.; Roy, A.; Beaupre, S.; Cho, S.; Coates, N.; Moon, J. S.; Moses, D.; Leclerc, M.; Lee, K.; Heeger, A. J. Bulk Heterojunction 
Solar Cells with Internal Quantum Efficiency Approaching 100\%. Nat. Photon. 2009, 3, 297-302.

(21) Chen, H.-Y.; Hou, J.; Zhang, S.; Liang, Y.; Yang, G.; Yang, Y.; Yu, L.; Wu, Y.; Li, G. Polymer Solar Cells with Enhanced Opencircuit Voltage and Efficiency. Nat. Photon. 2009, 3, 649-653.

(22) Stevens, A. L.; Kaeser, A.; Schenning, A. P. H. J.; Herz, L. M. MorphologyDependent Energy Transfer Dynamics in Fluorene-Based Amphiphile Nanoparticles. ACS Nano 2012, 6, 4777.

(23) Shaw, P. E.; Ruseckas, A.; Peet, J.; Bazan, G. C.; Samuel, I. D. W. ExcitonExciton Annihilation in Mixed-Phase Polyfluorene Films. Adv. Funct. Mater. 2010, 20, 155-161.

(24) Tretiak, S.; Saxena, A.; Martin, R. L.; Bishop, A. R. Conformational Dynamics of Photoexcited Conjugated Molecules. Phys. Rev. Lett. 2002, 89, 097402.

(25) Bouvier, B.; Dognon, J.-P.; Lavery, R.; Markovitsi, D.; Millié, P.; Onidas, D.; Zakrzewska, K. Influence of Conformational Dynamics on the Exciton States of DNA Oligomers. J. Phys. Chem. B 2003, 10\%, $13512-13522$.

(26) Montgomery, N. A.; Denis, J.-C.; Schumacher, S.; Ruseckas, A.; Skabara, P. J.; Kanibolotsky, A.; Paterson, M. J.; Galbraith, I.; Turnbull, G. A.; Samuel, I. D. W. Optical Excitations in Star-Shaped Fluorene Molecules. J. Phys. Chem. A 2011, 115, 2913-2919.

(27) Lin, H.; Tabaei, S. R.; Thomsson, D.; Mirzov, O.; Larsson, P.-O.; Scheblykin, I. G. Fluorescence Blinking, Exciton Dynamics, and Energy Transfer Domains in Single Conjugated Polymer Chains. Journal of the American Chemical Society 2008, 130, 7042-7051.
(28) Cao, J.; Silbey, R. J. Optimization of Exciton Trapping in Energy Transfer Processes. J. Phys. Chem. A 2009, 113, 13825-13838.

(29) Athanasopoulos, S.; Emelianova, E. V.; Walker, A. B.; Beljonne, D. Exciton Diffusion in Energetically Disordered Organic Materials. Phys. Rev. B 2009, 80, 1-7.

(30) Meng, L.; Shang, Y.; Li, Q.; Li, Y.; Zhan, X.; Shuai, Z.; Kimber, R. G. E.; Walker, A. B. Dynamic Monte Carlo Simulation for Highly Efficient Polymer Blend Photovoltaics. J. Phys. Chem. B 2010, 114, 36-41.

(31) Daniel, C.; Westenhoff, S.; Makereel, F.; Friend, R. H.; Beljonne, D.; Herz, L. M.; Silva, C. Monte Carlo Simulation of Exciton Bimolecular Annihilation Dynamics in Supramolecular Semiconductor Architectures. J. Phys. Chem. C 2007, 111, 19111-19119.

(32) Watkins, P. K.; Walker, A. B.; Verschoor, G. L. B. Dynamical Monte Carlo Modelling of Organic Solar Cells: The Dependence of Internal Quantum Efficiency on Morphology. Nano Lett. 2005, 5, 1814 1818, PMID: 16159229.

(33) Bednarz, M.; Malyshev, V. A.; Knoester, J. Intraband Relaxation and Temperature Dependence of the Fluorescence Decay Time of One-dimensional Frenkel Excitons: The Pauli Master Equation Approach. J. Chem. Phys. 2002, 117, 6200-6213.

(34) Takahata, M.; Nakano, M.; Fujita, H.; Yamaguchi, K. Mechanism of Exciton Migration of Dendritic Molecular Aggregate: a Master Equation Approach Including Weak Exciton-phonon Coupling. Chem. Phys. Lett. 2002, 363, 422-428.

(35) Nakano, M.; Takahata, M.; Yamada, S.; Yamaguchi, K.; Kishi, R.; Nitta, T. Exciton Migration Dynamics in a Dendritic 
Molecule: Quantum Master Equation Approach Using $\mathrm{Ab}$ Initio Molecular Orbital Configuration Interaction Method. $J$. Chem. Phys. 2004, 120, 2359-2367.

(36) Schumacher, S.; Galbraith, I.; Ruseckas, A.; Turnbull, G. A.; Samuel, I. D. W. Dynamics of Photoexcitation and Stimulated Optical Emission in Conjugated Polymers: A Multiscale Quantum-chemistry and Maxwell-Blochequations Approach. Phys. Rev. B 2010, 81, 245407.

(37) Tozer, O. R.; Barford, W. Exciton Dynamics in Disordered Poly(pphenylenevinylene). 1. Ultrafast Interconversion and Dynamical Localization. $J$. Phys. Chem. A 2012, 116, 10310-10318.

(38) Hidayat, R.; Fujii, A.; Ozaki, M.; Teraguchi, M.; Masuda, T.; Yoshino, K. Exciton Dynamics in Disubstituted Polyacetylenes. Synth. Met. 2001, 119, 597598.

(39) Brütting, W. Physics of Organic Semiconductors; WILEY-VCH Verlag, 2004; Vol. 201; pp 1035-1035.

(40) Zhang, W. M.; Meier, T.; Chernyak, V.; Mukamel, S. Exciton-migration and Three-pulse Femtosecond Optical Spectroscopies of Photosynthetic Antenna Complexes. J. Chem. Phys. 1998, 108, 7763-7774.

(41) Groves, C.; Greenham, N. Monte Carlo Simulations of Organic Photovoltaics; Topics in Current Chemistry; Springer Berlin Heidelberg, 2013; pp 1-22.

(42) Mesta, M.; Carvelli, M.; de Vries Rein, J.; van Eersel, H.; van der Holst Jeroen, M. J.; Schober, M.; Furno, M.; Lüssem, B.; Leo, K.; Loebl, P. et al. Molecular-scale Simulation of Electroluminescence in a Multilayer White Organic Light-emitting Diode. Nat. Mater. 2013, 12, 652-658.

(43) Bässler, H. Charge Transport in Disordered Organic Photoconductors a Monte
Carlo Simulation Study. Phys. Status Solidi B 1993, 175, 15-56.

(44) Meskers, S. C. J.; Hübner, J.; Oestreich, M.; Bässler, H. Dispersive Relaxation Dynamics of Photoexcitations in a Polyfluorene Film Involving Energy Transfer: Experiment and Monte Carlo Simulations. J. Phys. Chem. B 2001, 105, 9139-9149.

(45) Kimber, R. G. E.; Wright, E. N.; O'Kane, S. E. J.; Walker, A. B.; Blakesley, J. C. Mesoscopic Kinetic Monte Carlo Modeling of Organic Photovoltaic Device Characteristics. Phys. Rev. B 2012, 86, 235206.

(46) Mendels, D.; Tessler, N. Drift and Diffusion in Disordered Organic Semiconductors: The Role of Charge Density and Charge Energy Transport. J. Phys. Chem. C 2013, 117, 3287-3293.

(47) Soutar, I.; Swanson, L.; Imhof, R. E.; Rumbles, G. Synchrotron-generated Time-resolved Fluorescence Anisotropy Studies of the Segmental Relaxation of Poly(acrylic acid) and Poly(methacrylic acid) in Dilute Methanolic Solutions. Macromolecules 1992, 25, 4399-4405.

(48) Montgomery, N. A.; Hadley, G.; Ruseckas, A.; Denis, J.-C.; Schumacher, S.; Kanibolotsky, A. L.; Skabara, P. J.; Galbraith, I.; Turnbull, G. A.; Samuel, I. D. W. Dynamics of Fluorescence Depolarization in Branched Oligofluorene-truxene Molecules. Phys. Chem. Chem. Phys. 2012, 14, 9176.

(49) Andrews, D. L.; Curuchet, C.; Scholes, G. D. Resonance Energy Tranfer: Beyond the Limits. Laser Photonics Rev. 2011, 5, 114.

(50) Olaya-Castro, A.; Scholes, G. D. Energy Transfer from Förster-Dexter Theory to Quantum Coherent Light-harvesting. Int. Rev. Phys. Chem. 2011, 30, 49-77. 
(51) Beenken, W. J. D.; Pullerits, T. O. Excitonic Coupling in Polythiophenes: Comparison of Different Calculation Methods. J. Chem. Phys. 2004, 120, 2490-2495.

(52) Westenhoff, S.; Daniel, C.; Friend, R. H.; Silva, C.; Sundström, V.; Yartsev, A. Exciton Migration in a Polythiophene: Probing the Spatial and Energy Domain by Line-dipole Forster-type Energy Transfer. J. Chem. Phys. 2005, 122, 094903.

(53) Denis, J.-C.; Schumacher, S.; Galbraith, I. Quantitative Description of Interactions Between Linear Organic Chromophores. J. Chem. Phys. 2012, 137, 224102.

(54) Schumacher, S.; Ruseckas, A.; Montgomery, N. A.; Skabara, P. J.; Kanibolotsky, A. L.; Paterson, M. J.; Galbraith, I.; Turnbull, G. A.; Samuel, I. D. W. Effect of Exciton Self-trapping and Molecular Conformation on Photophysical Properties of Oligofluorenes. J. Chem. Phys. 2009, 131, 154906 .

(55) Crank, J. In The Mathematics of Diffusion; Press, O. C., Ed.; Oxford: Clarendon Press, 1956.

(56) Wu, J.; Berland, K. M. Propagators and Time-Dependent Diffusion Coefficients for Anomalous Diffusion. Biophys. J. 2008, 95, $2049-2052$.

(57) Lyons, B. P.; Monkman, A. P. The Role of Exciton Diffusion in Energy Transfer Between Polyfluorene and Tetraphenyl Porphyrin. Phys. Rev. B 2005, 71, 235201.

(58) Richter, M.; Renger, T.; Renger, G.; Knorr, A. Nonperturbative Theory for the Optical Response to Strong Light of the Light Harvesting Complex II of Plants: Saturation of the Fluorescence Quantum Yield. J. Chem. Phys. 2007, 127, 075105.

(59) Ling, S.; Schumacher, S.; Galbraith, I.; Paterson, M. J. Excited-State Absorption of Conjugated Polymers in the NearInfrared and Visible: A Computational
Study of Oligofluorenes. J. Phys. Chem.

C 2013, 117, 6889-6895. 


\section{Graphical TOC Entry}

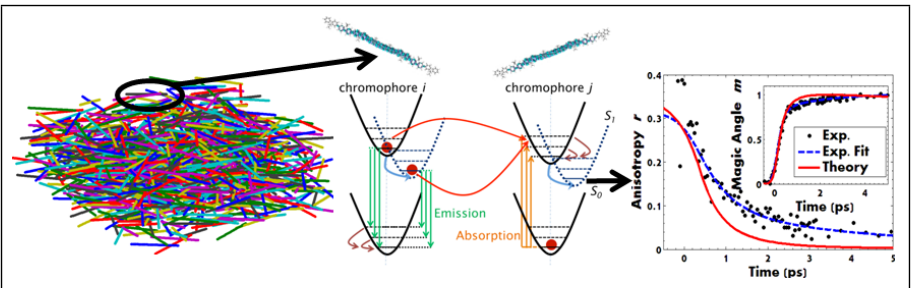

Sketch of our model for incoherent exciton transfer and film morphology which predicts well the observed ultrafast anisotropy decay. 